\title{
Prospective impact of 5-FU in the induction of endoplasmic reticulum stress, modulation of GRP78 expression and autophagy in Sk-Hep1 cells
}

\author{
ANANDAM KASIN YADUNANDAM ${ }^{1}$, JIN-SOO YOON ${ }^{1}$, YEONG-AE SEONG $^{1}$, \\ CHUL-WOONG $\mathrm{OH}^{2}$ and GUN-DO KIM ${ }^{1}$ \\ ${ }^{1}$ Department of Microbiology, College of Natural Sciences, ${ }^{2}$ Department of Marine Biology, \\ College of Fishery Sciences, Pukyong National University, Busan 608-737, Republic of Korea
}

Received March 8, 2012; Accepted April 27, 2012

DOI: 10.3892/ijo.2012.1506

\begin{abstract}
Hepatocellular carcinoma (HCC) is one of the most aggressive malignant diseases and is highly resistant to conventional chemotherapy. Therefore, HCC requires more effective prevention and treatment strategies. 5-fluorouracil (5-FU) remains the most widely used chemotherapeutic drug for the treatment of gastrointestinal, breast, head and neck, and ovarian cancers. In pursuit of a novel effective strategy, we have evaluated the potential of 5-FU to promote endoplasmic reticulum (ER) stress and autophagy in Sk-Hep1 HCC cells. We found that 5-FU profoundly induces ER stress in Sk-Hep1 cells and upregulates p53 and activates CHOP/ GADD153 and caspase-12. Activation of CHOP/GADD153 and caspase-12 promotes mitochondrial cell death in Sk-Hep1 cells followed by ER stress. Changes in calcium homeostasis and the protein folding machinery cause stress in the ER, leading to apoptotic cell death. Stress in the ER activates autophagy to remove the misfolded protein aggregates and recover from the stress environment. Our study demonstrates that 5-FU-induced ER stress suppresses autophagy and also downregulates GRP78 expression. Activation of autophagy followed by ER stress facilitates the cell survival response. Therefore, the inhibition of protective autophagy may provide a useful pharmacological target. Taken together, these results indicate that 5-FU-induced ER stress activates the mitochondrial apoptotic cell death pathway by downregulating GRP78
\end{abstract}

Correspondence to: Professor Gun-Do Kim, Department of Microbiology, College of Natural Sciences, Pukyong National University, 599-1, Daeyeon 3-Dong, Nam-Gu, Busan 608-737, Republic of Korea

E-mail: gundokim@pknu.ac.kr

Abbreviations: HCC, hepatocellular carcinoma; EMEM, Eagle's minimal essential medium; FBS, fetal bovine serum; WST-1, 2-(4-iodophenyl)-3-(4-nitrophenyl)-5-(2,4-disulfophenyl)-2Htetrazolium, monosodium salt; PBS, phosphate-buffered saline

Key words: hepatocellular carcinoma, 5-fluorouracil, ER stress, apoptosis, autophagy and protective autophagy proteins in Sk-Hep1 cells, raising the possibility of using 5-FU as a therapeutic agent to target human HCC.

\section{Introduction}

Hepatocellular carcinoma (HCC) is one of the most aggressive human types of cancer in the world and the second leading cause of cancer mortality; with few curative treatment options by surgical resection and/or transplantation. Current predictions suggest that new cases are increasing steeply, rising to 748,300 , with 695,900 deaths every year (1). This is due to the fact that only $15 \%$ of patients qualify for tumor resection and patients who undergo hepatectomy are reported to develop new tumors in their residual liver due to the aggressive features of HCC, which include poor liver function and metastasis (2). However, no major advances have been made in the past decades. Therefore, HCC remains a serious problem and more effective prevention and treatment strategies are urgently needed.

5-fluorouracil (5-FU), a pyrimidine based analog, has been widely used as an anticancer drug for the treatment of gastrointestinal, breast, head and neck, and ovarian cancers. Due to its clastogenic nature, 5-FU is converted to 5-fluoro-2-deoxyuridylate monophosphate (FdUMP), and it competitively inhibits DNA synthesis by inhibiting the enzyme thymidylate synthase $(3,4)$. 5-FU induced DNA damage activates various signaling pathways to enhance or inhibit apoptotic cell death. 5-FU by itself or in conjunction with andrographolide induces apoptosis and inhibition of cell proliferation through a p53 mediated pathway in human colon cancer cells $(5,6)$.

Apoptosis is a form of cell death that involves the consecutive action of a number of intracellular signaling pathways. Resistance to apoptotic induction is an important cause of failure in chemotherapy; therefore, strengthening apoptosis induction might improve the effect of anticancer therapy. It has been suggested that there are several apoptotic pathways in cells responsive to death stimuli. Previous studies $(7,8)$ have concentrated on the endoplasmic reticulum (ER) as a third subcellular compartment implicated in apoptotic execution. Accumulation of misfolded proteins and changes in $\mathrm{Ca}^{2+}$ 
homeostasis in ER results in ER stress and eventually leads to apoptotic cell death (7). In general, autophagy can activate both pro-survival mechanisms as well as cell death programs, especially if autophagy activated following ER stress is a prosurvival response to restore the ER homeostasis by clearing the unfolded aggregates (8). A wide range of chemotherapeutic agents have been identified and are used to activate ER stress along with autophagy in carcinoma cells (8). Heat shock proteins function as molecular chaperones in regulating cellular homeostasis and promote cell survival response. Inhibition of autophagy and molecular chaperones might be a suitable pharmacological target to promote apoptosis in tumor cells. In this study, we have evaluated the effect of 5-FU to elicit ER stress-induced apoptosis in Sk-Hep1 cells. The results suggest that 5-FU induces ER stress and activates the intrinsic apoptotic cell death pathway by downregulating GRP78 and autophagy in hepatocarcinoma Sk-Hep1 cells, explaining a multiple role of 5-FU as a therapeutic agent targeting human HCC.

\section{Materials and methods}

Cell culture and reagents. Human Sk-Hep1 cells (American Type Culture Collection, Manassas, VA, USA) were cultured in EMEM supplemented with 10\% FBS (v/v) (HyClone, Logan, UT, USA) and penicillin (100 U/ml)/streptomycin $(100 \mu \mathrm{g} / \mathrm{ml})$ (PAA Laboratories $\mathrm{GmbH}$, Pasching, Austria). Cultures were maintained in a humidified incubator at $37^{\circ} \mathrm{C}$ in $5 \% \mathrm{CO}_{2} .5-\mathrm{FU}$, dimethyl sulfoxide and propidium iodide were purchased from Sigma Aldrich (St. Louis, MO, USA). EZ-Cytox Cell Viability Assay Solution (WST-1) was purchased from Daeil Lab Service (Jong-No, Seoul, Korea). Lysis buffer was obtained from Intron Biotechnology (Gyeonggi, Korea). Hoechst 33342 was purchased from Cell Signaling Technology (Danvers, MA, USA) and Fluo3-AM was from Invitrogen (Grand Island, NY, USA). Enhanced chemiluminescent (ECL) detection solutions were obtained from Pierce (Rockford, IL, USA). Anti-HSP70, anti-HSP27, anti-CHOP/GADD153, and antiAIF were obtained from Santa Cruz Biotechnology, Inc (Santa Cruz, CA, USA). All other antibodies were obtained from Cell Signaling Technology.

Cell viability assay. Sk-Hep1 cells in exponential growth phase at a density of $1 \times 10^{4}$ cells were resuspended in $100 \mu \mathrm{l}$ of EMEM medium and seeded on 96-well plates in triplicate. Following overnight incubation, 5-FU at various concentrations was added. Cells were incubated for $24 \mathrm{~h}$, and $10 \mu \mathrm{l}$ of WST-1 solution was added and incubated for an additional $3 \mathrm{~h}$. The absorbance of the reaction was measured using an ELISA reader (Molecular Devices, Sunnyvale CA, USA) at $460 \mathrm{~nm}$. Cell viability was calculated according to the following formula: Cell viability $(\%)=\mathrm{A} 460($ sample$) / \mathrm{A} 460($ control $) \times 100$.

In situ labeling of apoptotic cells. Cells were cultured in a cover glass bottom culture dish for $24 \mathrm{~h}$ and treated with graded concentrations of 5-FU for another $24 \mathrm{~h}$. Following incubation, cells were washed twice with PBS, stained with Hoechst 33342 $(1 \mu \mathrm{g} / \mathrm{ml})$ for $15 \mathrm{~min}$ at $37^{\circ} \mathrm{C}$, and then fixed for $15 \mathrm{~min}$ with $4 \%$ paraformaldehyde. The cells were examined under an ECLIPSE 50i fluorescence microscope (Nikon, Tokyo, Japan).
Flow cytometry. Briefly, cells were harvested by trypsinization and fixed with $70 \%$ ethanol overnight at $4^{\circ} \mathrm{C}$. Then, the cells were resuspended in PBS buffer containing $0.2 \mathrm{mg} / \mathrm{ml}$ RNase A and incubated for $1 \mathrm{~h}$ at $37^{\circ} \mathrm{C}$. The cells were then stained with $40 \mu \mathrm{g} / \mathrm{ml}$ propidium iodide at room temperature for $30 \mathrm{~min}$ under dark conditions. The distribution of subgenomic DNA was analyzed using a flow cytometer (Becton-Dickinson, Franklin Lakes, NJ, USA).

Western blot analysis. After treatment with 5-FU, cells were washed twice with ice-cold PBS and the reaction was terminated by the addition of $100 \mu \mathrm{l}$ lysis buffer containing $50 \mathrm{mM}$ Tris-Cl (pH 7.5), $150 \mathrm{mM} \mathrm{NaCl}, 1$ mM DTT, 0.5\% NP-40, 1\% Triton X-100, $1 \%$ deoxycholate, $0.1 \%$ SDS, and protease inhibitors (PMSF, EDTA, aprotinin, leupeptin, prostatin A). Western blotting was performed using $30 \mu \mathrm{g}$ of protein separated by electrophoresis in a $12 \%$ polyacrylamide gel, transferred to a nitrocellulose membrane, and immuno-reacted with the indicated antibodies.

Fluo3-AM calcium assay. Cells were cultured in a cover glass bottom culture dish for $24 \mathrm{~h}$, treated with graded concentrations of 5-FU for another $24 \mathrm{~h}$, and then stained with Fluo3-AM $(1.5 \mu \mathrm{M})$ for $30 \mathrm{~min}$ at $37^{\circ} \mathrm{C}$. Cells were washed twice with PBS and examined under an Eclipse 50i fluorescence microscope (Nikon).

Immunofluorescence. Cells grown on a cover glass bottom culture dish were treated with 5-FU, washed with PBS, fixed with $4 \%$ paraformaldehyde for $15 \mathrm{~min}$ at room temperature, and then blocked for $1 \mathrm{~h}$ in PBS containing 0.3\% Triton X-100. Then, the cells were stained with 1:800 diluted anti-p53 and anti- $\beta$-actin polyclonal antibodies, and incubated for $16 \mathrm{~h}$ at $4^{\circ} \mathrm{C}$. Cells were washed with PBS and incubated for $1 \mathrm{~h}$ with Alexa Fluor-488-conjugated secondary antibody and Alexa Fluor-555-conjugated secondary antibody (Cell Signaling Technology). To visualize the nucleus, cells were counterstained with $1 \mu \mathrm{g} / \mathrm{ml}$ DAPI (Roche, Pleasanton, CA, USA). After mounting, the cells were analyzed with an Eclipse 50i fluorescence microscope.

\section{Results}

5-FU treatment results in apoptotic cell death of Sk-Hepl cells. A dose response study was conducted to investigate the effect of 5-FU on the growth of Sk-Hep1 HCC cells. As shown in Fig. 1A, 5-FU inhibited Sk-Hep1 cell proliferation after $24 \mathrm{~h}$ exposure, with an $\mathrm{IC}_{50}$ value of $50 \mu \mathrm{g} / \mathrm{ml}$. In order to determine the mode of cell death induced by 5 -FU, we examined the nuclear morphology of cells using Hoechst 33342, a DNA-specific fluorescent dye. When cells were treated with $50 \mu \mathrm{g} / \mathrm{ml}$ 5-FU for $24 \mathrm{~h}$, the Sk-Hep1 cells exhibited condensed and fragmented nuclei similar to apoptotic cell morphology (Fig. 1B), indicating that 5-FU induces apoptotic cell death in Sk-Hep1 HCC cells.

5-FU induces ER stress in Sk-Hepl cells. To determine whether 5-FU has possible effects on ER, several ER-specific mediators were analysed. Initially, we focused on the expression levels of caspase-12 and caspase-7, using respective antibodies, 

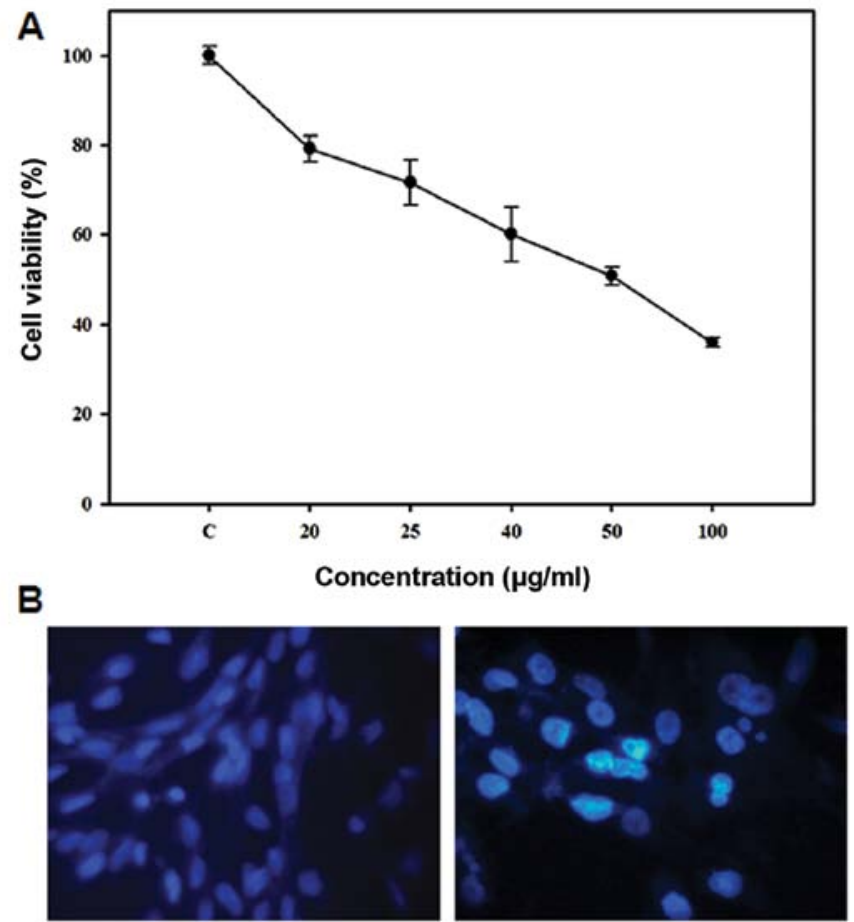

Figure 1. Identification of 5-FU-induced antitumor effect in Sk-Hep1 cells (A) Cells were incubated with different concentrations of 5-FU for $24 \mathrm{~h}$. Cell viability was measured by WST- 1 cell proliferation assay. $\mathrm{IC}_{50}$ was calculated as described in the Materials and methods. (B) Cells were cultured for $24 \mathrm{~h}$ and then treated with $50 \mu \mathrm{g} / \mathrm{ml}$ of $5-\mathrm{FU}$ for an additional $24 \mathrm{~h}$. After incubation, the cells were washed and stained with Hoechst 33342 and then observed under a fluorescence microscope.

and the results showed that activation occurred from $24 \mathrm{~h}$ of 5-FU treatment (Fig. 2A). This suggests that cleavages of these two caspases are palpable in ER stress. We next examined the expression of CHOP/GADD153, a transcription factor induced by cellular stresses such as UV light and ER stress (9). The expression of CHOP/GADD153 was dramatically increased in a time-dependent manner by 5-FU treatment (Fig. 2A). These data indicate that 5-FU induces ER stress and it may initiate apoptotic cell death in Sk-Hepl cells.

As depletion of $\mathrm{Ca}^{2+}$ homeostasis in the ER lumen can modify numerous cellular responses, and especially accumulation of misfolded proteins. The mobilization of intracellular $\mathrm{Ca}^{2+}$ was examined by Fluo3-AM (a green fluorescent $\mathrm{Ca}^{2+}$ sensor). This analysis shows that 5-FU increases the intracellular $\mathrm{Ca}^{2+}$ mobilization in a time-dependent manner (Fig. 2B). Moreover, the activation of calpain, an indicator of $\mathrm{Ca}^{2+}$ homeostasis disruption, was also detected in its active form of about $75 \mathrm{kDa}$ products (Fig. 2C).

ER stress enhances the expression of p53 and bcl-2 family proteins. The tumor suppressor 553 plays a pivotal role in cells by enhancing apoptotic genes and inducing inhibition of the cell cycle in response to various forms of stress. It has been shown that ER stress induces increased accumulation of p53 in MEF (10). On the other hand, p53 activity was inhibited in ER stressed WI-38 and HT1080 cells (11). To identify the expression levels of p53 in ER under stressed conditions, Sk-Hep1 cells were treated with 5-FU and ER stress was found

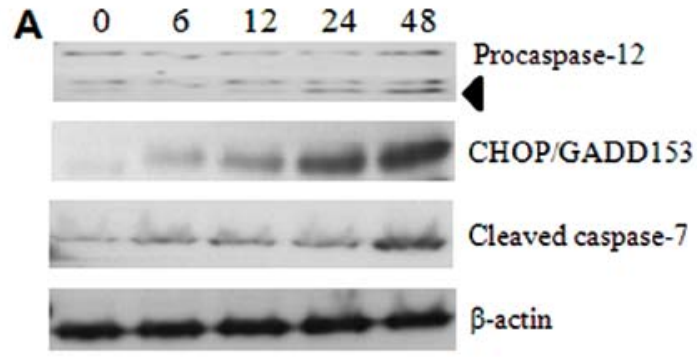

B
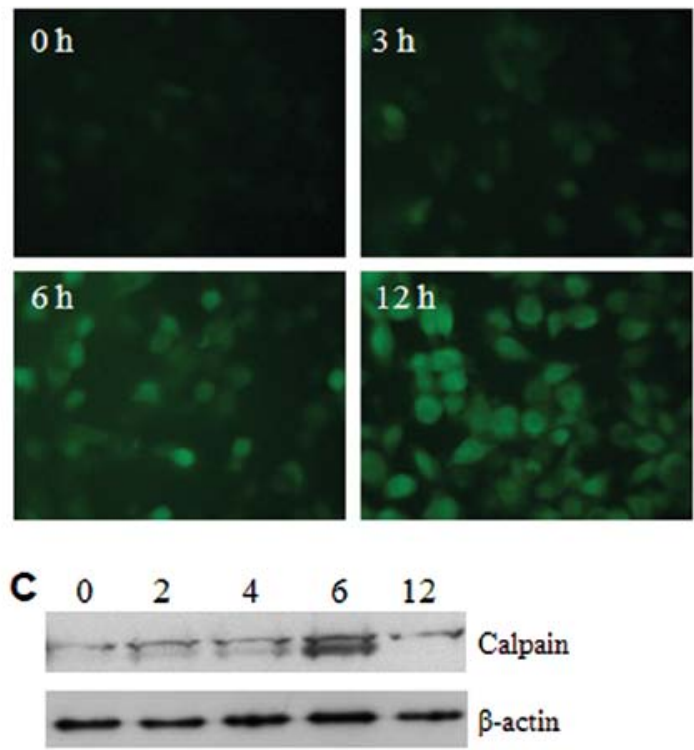

Figure 2. Induction of ER stress mediators, $\mathrm{Ca}^{2+}$ mobilization and calpain activation in 5-FU-treated Sk-Hep1 cells. (A) Cells were incubated with $50 \mu \mathrm{g} / \mathrm{ml}$ of $5-\mathrm{FU}$ for $6,12,24$, and $48 \mathrm{~h}$. Then, the cells were harvested and lysed for the detection of caspase-7, -12 and CHOP/GADD153. The arrow head indicates the cleaved form of caspase-12. After the treatment with 5-FU for the indicated times, (B) the cells were stained with Fluo3-AM for detection of intracellular $\mathrm{Ca}^{2+}$ mobilization, or (C) harvested and lysed for the detection of cleavage and activation of calpain by western blot analysis.

to induce large changes in the expression pattern of p53 within 6-12 h in Sk-Hep1 cells (Fig. 3A). To further investigate the nuclear retention of $\mathrm{p} 53$, the 5-FU exposed Sk-Hep1 cells were subjected to immunofluorescence studies. The results showed that p53 was intact in the nucleus (Fig. 3B), thus substantiating that 5-FU-induced ER stress promotes nuclear accumulation and stabilization of this tumor suppressor protein in hepatoma cells.

The Bcl-2 family proteins PUMA and Noxa are targets of p53 and are involved in ER stress-induced apoptosis (12). Recent evidence reveals that Bax and Bak can also be localized in ER and activated in response to ER stress (13). Western blotting was used to test whether the induction of a subset of Bcl-2 family proapoptotic proteins in 5-FU induced ER stress, and we observed that p53 targeted proteins (PUMA and Noxa) and Bak were increased time-dependently in Sk-Hep1 cells, whereas Bax expression remains unchanged (Fig. 3C). Taken together, these findings demonstrate that in Sk-Hep1, induction of ER stress by 5-FU causes overexpression, and nuclear stabilization of $\mathrm{p} 53$ promotes the expression of proapoptotic Bcl-2 family proteins. 

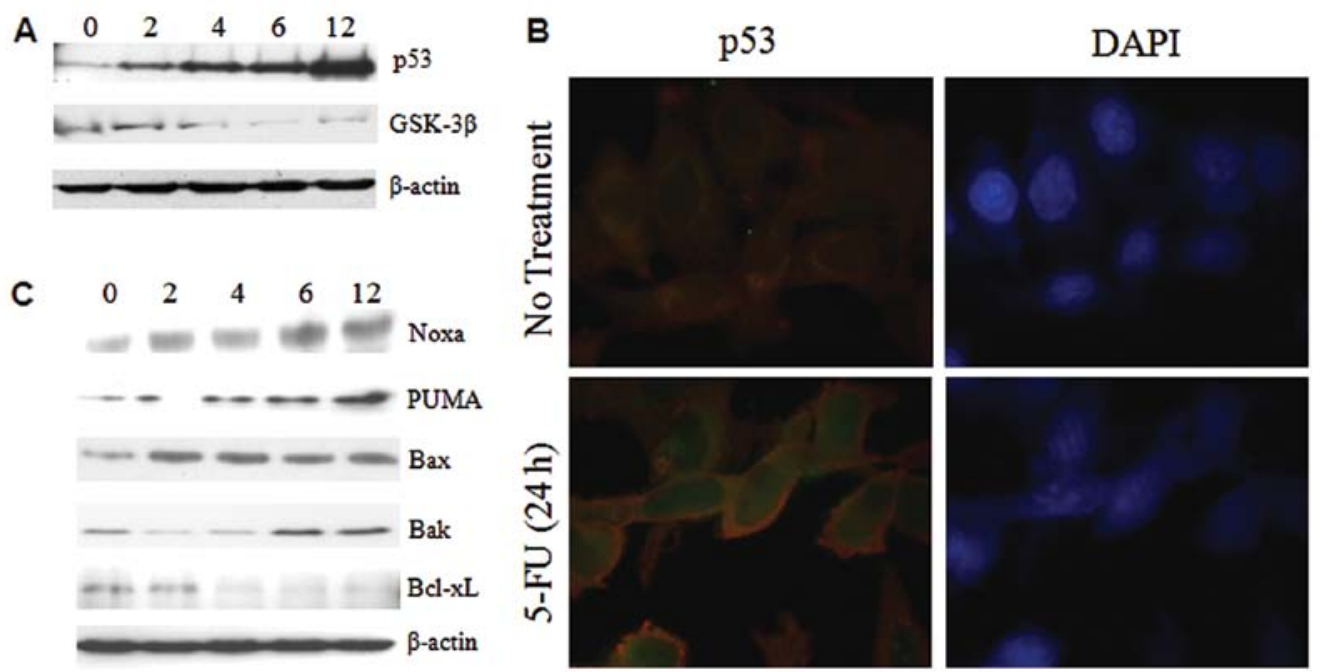

Figure 3. Regulation of p53 and Bcl-2 family member proteins by 5-FU-induced ER stress in Sk-Hepl cells. (A) Cells were treated with $50 \mu \mathrm{g} / \mathrm{ml}$ of 5-FU for the indicated time course and harvested. Cell extracts were lysed and subjected to immunoblotting with anti-p53 and anti-GSK-3 $\beta$ antibodies. (B) 5 -FU treated Sk-Hep1 cells were subjected to immunostaining for upregulated p53 (green). The nuclear retention of p53 was detected by fluorescence microscopy. (C) Cells were treated with $50 \mu \mathrm{g} / \mathrm{ml}$ of 5-FU for the indicated times. Cell lysates were subjected to western blot analysis and immuno-reacted with proapoptotic antibodies.
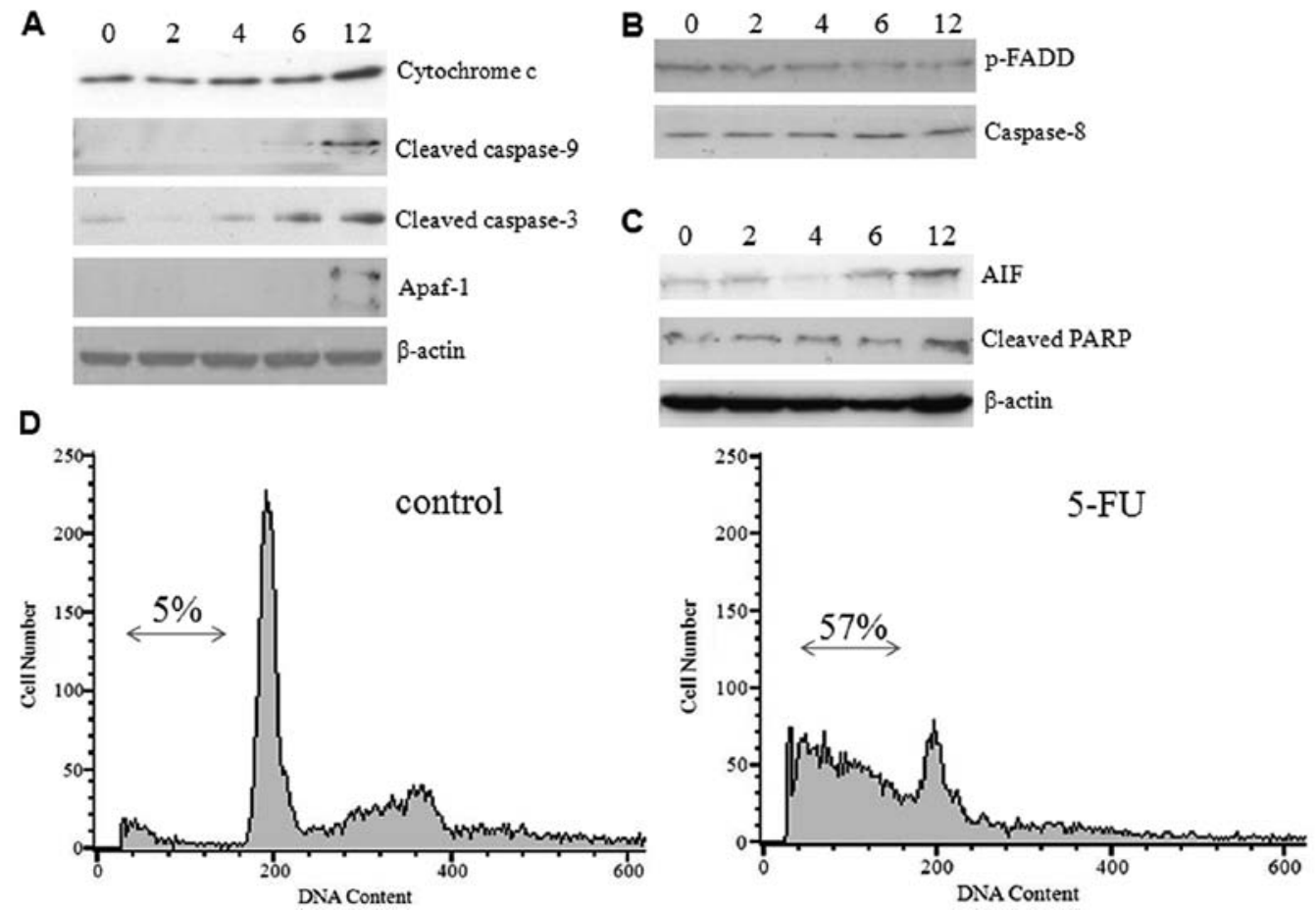

Figure 4. Expressions of caspases and other apoptogenic proteins in ER stressed cells. Cells were incubated with $50 \mu \mathrm{g} / \mathrm{ml}$ of 5 -FU for the indicated times. Proteins $(30 \mu \mathrm{g})$ were separated by electrophoresis in 12\% polyacrylamide gel, transferred to a nitrocellulose membrane and immuno-reacted with caspases and other pro- and anti-apoptotic antibodies (A, B and C). Quantification of sub-G1 DNA content and cell cycle arrest in Sk-Hep1 cells treated with 5-FU for $24 \mathrm{~h}$ were analyzed by flow cytometry $(\mathrm{C})$.

ER stress activates the intrinsic mitochondrial pathway. The transition of ER stress to an apoptotic response is not clearly understood, but it does appear to be dependent on caspases and proteins of the Bcl-2 family. The release of cytochrome $c$ from mitochondria and activation of initiator caspase-9 which occurred during ER stress-induced apoptosis suggest the involvement of an intrinsic cell death pathway (14). To investigate whether 5-FU-induced ER stress causes the release of cytochrome $c$ and other apoptogenic proteins from mitochondria, we examined the expression levels of caspases and proapoptotic proteins by western blot analysis using commercially available antibodies. Increased protein expression was detected for cytochrome $c$, cleaved caspase-9, and Apaf-1 (molecular core of the apoptosome) in a time-dependent manner. The effector caspase- 3 was found in active form in a gradient manner (Fig. 4A). In contrast, the expression of 

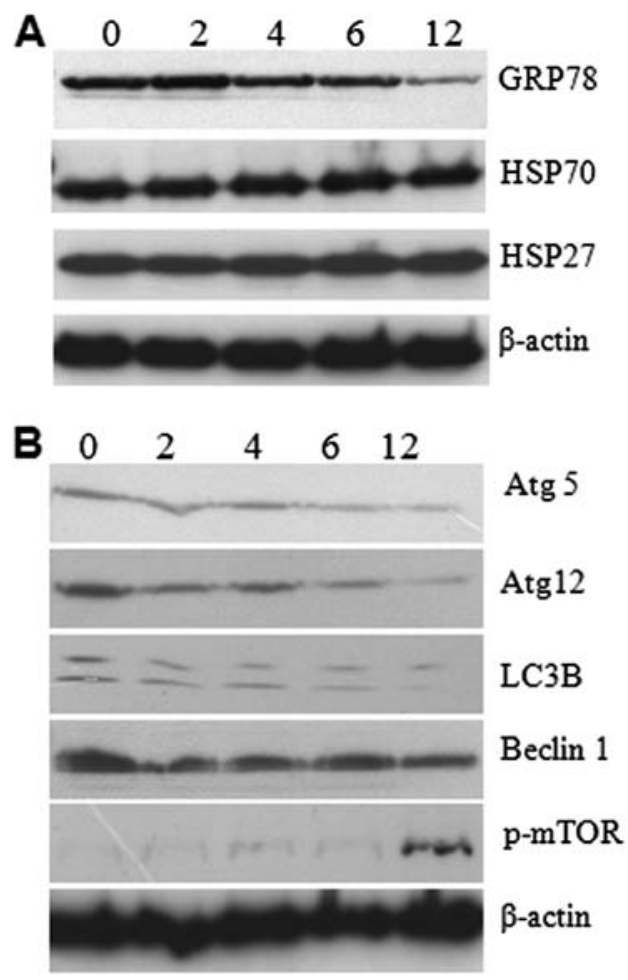

Figure 5. Modulated expression of GRP78 and autophagy proteins in Sk-Hep1 cells. Cells were incubated with $50 \mu \mathrm{g} / \mathrm{ml}$ of 5 -FU for the indicated times. Proteins $(30 \mu \mathrm{g})$ were separated by electrophoresis in a $12 \%$ polyacrylamide gel, transferred to a nitrocellulose membrane and immuno-reacted with antiGRP78 (A) and autophagy antibodies (B).

phospho-FADD and caspase- 8 were decreased gradually (Fig. 4B) suggesting that 5-FU-induced ER stress did not activate the death receptor pathway. The hepatoma cells exposed to 5-FU expressed significant amounts of cleaved PARP and AIF (Fig. 4C), thus confirming programmed cell death, which was previously observed (15). These results confirm that 5-FU-induced ER stress promotes the mitochondrial cell death pathway in Sk-Hep1 cells by over-expressing the proapoptotic proteins. To further substantiate our results, we examined the effect of 5-FU-induced cell death by FACS analysis and demonstrated a higher population of Sk-Hep1 cells in the sub-G1 phase $(\sim 57 \%)$, as opposed to the control cells $(\sim 5 \%)$ (Fig. 4D). We also noted that treatment of Sk-Hep1 cells with 5-FU was able to elicit a G1/S arrest (Fig. 4D).

ER stress downregulates the expression of GRP78 and autophagy. Glucose regulated protein 78 (GRP78) is an ER chaperone protein with $\mathrm{Ca}^{2+}$ binding and antiapoptotic properties; it is required for protein folding, assembly of membrane and secretary proteins, and to mitigate ER stress. It has been shown that GRP78 protect tumor cells and prevent apoptosis by interfering with caspase activation in many different cell types in response to a variety of cellular stresses and a range of chemotherapeutic agents $(16,17)$. Autophagy is a process of degradation of long-lived proteins and sub-cellular organelles through lysosomal machinery, frequently activated in response to an adverse environment or stress (8). Accumulation of terminally misfolded protein aggregates in ER can be effectively removed by autophagy (18). To better understand the

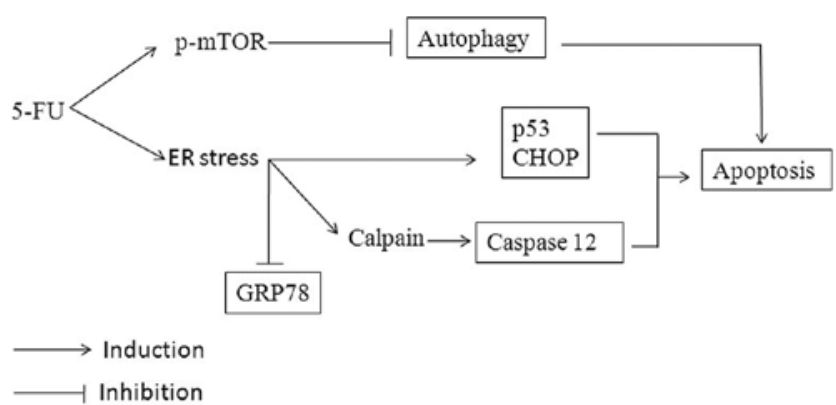

Figure 6. Interrelationship between ER and the mitochondrial signaling pathway. ER stress activates caspase-12,p53, PUMA, and Noxa. As members of the Bcl-2 protein family, PUMA and Noxa induce release of cytochrome $c$ from mitochondria through activation of Bax and Bak. Cytochrome $c$ binds to caspase- 9 and Apaf-1 to form an apoptosome. Caspase-12 also activates caspase-9, which then activates effector caspases-3 and -7, to induce cell death. ER stress induces CHOP/GADD153 that may also contribute to ER stress-induced apoptosis. Inhibition of GRP78 and autophagy may induce apoptotic cell death.

5-FU-induced ER stress and its consequent intrinsic apoptosis, we administered 5-FU to the Sk-Hep1 cells and performed western blot analysis for GRP78 and autophagy proteins. The results showed that expressions of GRP78 decreased gradually, which explains how prolonged ER stress can cause failure in the ER recovery process and lead to cell death (Fig. 5A). In addition to GRP78, we studied the expression level of heat shock proteins by western analysis. The result reveals no changes in the expression level suggesting that 5-FU-induced ER stress did not affect the expression of heat shock proteins (Fig. 5A). The autophagy related LC3, Atg5, Atg12, and Beclin1 expressions were gradually decreased in a timedependent manner (Fig. 5B). In contrast, we found enhanced phospho-mTOR in 5-FU-treated cells with optimum expression at $12 \mathrm{~h}$ of exposure (Fig. 5B). It seems that the enhanced phospho-mTOR resulted in the downregulation of autophagyrelated LC3, Atg5, Atg12, and Beclin1 expression. It suggests that downregulation of the expression of autophagy-related proteins promote apoptosis in Sk-Hepl cells due to accumulation of misfolded protein aggregates.

\section{Discussion}

The key focus of molecular cancer investigation is the development of new therapeutic strategies and the design of drugs to target the genetic and biochemical genesis of malignant transformation. To achieve this goal, many conventional and experimental chemotherapeutic drugs have been used to stimulate ER stress along with autophagy in cancer cells (8). Among the chemotherapeutic agents, 5-FU remains the most widely used drug and is used in many cancer treatments. In this study, we have shown that 5-FU-induced ER stress enhances the expression of p53 and Bcl-2 family proteins but downregulates the expression of GRP78 and autophagy related proteins. Based on the results, co-communications between 5-FU-induced ER stress and apoptosis are summarized in Fig. 6.

In this study, 5-FU profoundly induced the disruption of $\mathrm{Ca}^{2+}$ homeostasis and consequent expression of ER stress mediators. It has also been documented that $\mathrm{Ca}^{2+}$ elevation 
promotes mobilization of calpain to the ER surface, resulting in activation of caspase-12 (19). In addition, cleaved caspase-7 is suggested to be an upstream initiator caspase responsible for caspase-12 activation (14). As previously demonstrated, 5-FU induced $\mathrm{Ca}^{2+}$ mobilization and calpain expression indicates perturbation in $\mathrm{Ca}^{2+}$ homeostasis and ER stress activation. Furthermore, prolonged ER stress causes activation of caspase-7 and -12 , which indicates that both effectors are possibly attributed to ER stress-induced apoptosis. Consistent with prior reports $(20,21)$, the results showed that the expression level of CHOP/GADD153 was low at basal condition, but was elevated in response to 5-FU-induced ER stress (Fig. 2A), suggesting that prolonged stress initiates progression of apoptosis.

5-FU-induced ER stress increases the upregulation of Bcl-2 family proapoptotic (Bax, Bak, PUMA and Noxa) proteins, which indicates a possible crosstalk between ER and mitochondria. In addition, p53 expression was dramatically increased within $12 \mathrm{~h}$ following 5-FU treatment, suggesting that it might be due to the alteration in calcium homeostasis. Defects in nuclear retention of p53 inactivate its function in certain solid tumors, including hepatocellular carcinoma (22) and this inactivation is associated with metastasis and poor prognosis. It has been reported the inactivation of p53 by GSK-3 $\beta$ mediated phosphorylation in ER stressed cells rescues the cells from apoptosis. GSK-3 $\beta$ mediated p53 phosphorylation in the nucleus facilitating nucleocytoplasmic export and degradation (11). Here, 5-FU-induced ER stress decreased the activity and binding of GSK-3 $\beta$ to p53. Consistent with western blotting and immunofluorescence data (Fig. 3A and B), 5-FU-induced ER stress provokes nuclear retention of p53 by downregulating GSK-3 $\beta$. Activation of upstream ER stress modulators (CHOP and Bcl-2 family proteins) and effectors (caspase-12), ultimately direct to the activation of caspases, resulting in the sequential dismantling of the cell. Western blot analysis demonstrated increased cytosolic levels of cytochrome $c$, active initiator caspase-9, and Apaf-1 in 5-FU treated cells, suggesting that release of mitochondrial intermembrane space proteins into the cytosol forms apoptosomes in association with caspase-9 and Apaf-1. Furthermore, the expression of active downstream effector caspase-3 was also found to increase gradually. In this study, we determined increased levels of AIF and cleaved PARP, signifying that the active forms of AIF and PARP are involved in large scale DNA fragmentation and other cellular proteins (23). DNA fragmentation and apoptotic cell death were further confirmed by flow cytometry, and the sub-G1 content drastically increased up to $57 \%$ and cell cycle arrest was also observed. As confirmed by various reports, our data also ensures that Bcl-2 family proteins play a central role in regulating ER stress-induced apoptosis by promoting the release of apoptogenic factors $(10,12,13,24,25)$.

Prolonged ER stress causes initiation of cell death and the biochemical mechanism for the switch from an unfolded protein response (UPR) to apoptosis remains unclear. UPR provokes the induction of chaperone proteins which increases the protein folding capacity of the ER. The ER lumen houses a large array of molecular chaperones, among which, GRP78 is the best characterized and a member of the highly conserved HSP70 protein family. GRP78 can inhibit apoptosis by binding to caspase-7 and -12 . It acts as a $\mathrm{Ca}^{2+}$-binding protein to preserve $\mathrm{ER} \mathrm{Ca}^{2+}$ homeostasis and also as a chaperone to limit the aggregation of misfolded proteins $(16,26)$. Several studies have reported that GRP78 levels are increased in various types of cancer, including HCC (27). Increased expression of GRP78 enhances the protein folding capacity of ER and is associated with a pro-survival response. In fact, the diminished expression of GRP78 with siRNA activates the UPR and apoptosis in glioma and HeLa cells $(28,29)$. A lower level of GRP78 was associated with an increase in CHOP expression and activation of executioner caspases- 3 and -7 in a heterozygous mouse mammary model (30). The data presented here show that HCC constitutively overexpressed GRP78 and it was modulated by 5-FU-induced ER stress in Sk-Hep1 cells. This downregulation promotes the activation of caspase-7, -12 and CHOP/ GADD153, leading to apoptotic cell death.

This study also demonstrated that 5-FU-induced ER stress promotes apoptotic cell death by suppressing the protective autophagy in human HCC Sk-Hep1 cells. In general, ER stress and depletion of $\mathrm{Ca}^{2+}$ homeostasis induces the accumulation of misfolded proteins in the lumen. The accumulated unfolded proteins are refolded by eIF $2 \alpha$ through phosphorylation, or cleared by proteosomal degradation (31). Initial evidence suggests that autophagy could act as a potential degradation system for unfolded proteins accumulated in the ER (18). Recent reports have shown that autophagy is activated in response to ER stress for cell survival by degradation of both soluble and insoluble aggregates. Polyglutamine (poly-Q)induced ER stress activates autophagosome formation with LC3 conversion from LC3-I to LC3-II via PERK-dependent eIF $2 \alpha$ phosphorylation (31). Many ER stress inducing agents activate autophagy to eliminate polyubiquitinated unfolded protein aggregates in prostate and colon cancers, and autophagy induced by same chemicals does not confer resistance in normal colon cells and non-transformed murine embryonic fibroblasts (32). 5-FU-induced autophagy protects cells from apoptosis and deactivation by 3-methyladenine or Atg7 siRNA induces apoptosis in colon cancer cells $(33,34)$. Our study demonstrates that the 5-FU-induced ER stress promotes apoptotic cell death by suppressing the protective autophagy response in human HCC Sk-Hep1 cells.

In summary, it is suggested that 5-FU induces apoptotic signaling through a series of consecutive actions in Sk-Hep1 cells. It causes ER stress, which is characterized by the increase of CHOP/GADD153, p53 and caspase-12 activation, and $\mathrm{Ca}^{2+}$ mobilization. These expressions modulate GRP78 and autophagy activity, followed by interaction and activation of mitochondrial mediated apoptosis. The present study proposes that the induction of ER stress and downregulation of GRP78 and autophagy may be the major contributors for 5-FU-induced apoptosis in HCC Sk-Hep1 cells.

\section{Acknowledgements}

This work was supported by the Pukyong National University Research Abroad Fund in 2010 (PS-2010-0012000201004500). We thank Dr J. Venkatesan, Department of Chemistry, Pukyong National University, Korea, for his technical help during FACS analysis, and Dr P. Gopal and Dr T. Jebasingh (Madurai Kamaraj University, India) for their critical reading of our manuscript. 


\section{References}

1. Jemal A, Bray F, Center MM, Ferlay J, Ward E and Forman D: Global cancer statistics. CA Cancer J Clin 61: 69-90, 2011.

2. Roayaie S, Blume I, Thung S, et al: A system of classifying microvascular invasion to predict outcome after resection in patients with hepatocellular carcinoma. Gastroenterology 137: 850-855, 2009.

3. Sampath D, Rao V and Plunkett W: Mechanisms of apoptosis induction by nucleoside analogs. Oncogene 22: 9063-9074, 2003.

4. Longley D, Harkin D and Johnston P: 5-Fluorouracil: mechanisms of action and clinical strategies. Nat Rev Cancer 3: 330-338, 2003.

5. Yang L, Wu D, Luo K, Wu S and Wu P: Andrographolide enhances 5-fluorouracil-induced apoptosis via caspase-8-dependent mitochondrial pathway involving p53 participation in hepatocellular carcinoma (SMMC-7721) cells. Cancer Lett 276: 180-188, 2009.

6. Osaki M, Tatebe S, Goto A, Hayashi H, Oshimura M and Ito H 5-Fluorouracil (5-FU) induced apoptosis in gastric cancer cell lines: role of the p53 gene. Apoptosis 2: 221-226, 1997.

7. Rao R, Ellerby $\mathrm{H}$ and Bredesen D: Coupling endoplasmic reticulum stress to the cell death program. Cell Death Differ 11: 372-380, 2004

8. Tom V, Maria S, Guillermo V and Patrizia A: Linking ER stress to autophagy: potential implications for cancer therapy. Int J Cell Biol 2010: 930509, 2010.

9. Wang X, Lawson B, Brewer J, et al: Signals from the stressed endoplasmic reticulum induce $\mathrm{C} / \mathrm{EBP}$-homologous protein (CHOP/GADD153). Mol Cell Biol 16: 4273-4280, 1996.

10. Li J, Lee B and Lee A: Endoplasmic reticulum-stress induced apoptosis: Multiple pathways and activation of PUMA and NOXA by p53. J Biol Chem 281: 7260-7270, 2006.

11. Qu L, Huang S, Baltzis D, et al: Endoplasmic reticulum stress induces 553 cytoplasmic localization and prevents $\mathrm{p} 53$-dependent apoptosis by a pathway involving glycogen synthase kinase-3. Genes Dev 18: 261-277, 2004.

12. Shibue T, Suzuki S, Okamoto H, et al: Differential contribution of Puma and Noxa in dual regulation of p53-mediated apoptotic pathways. EMBO J 25: 4952-4962, 2006.

13. Zong W, Li C, Hatzivassiliou G, et al: Bax and Bak can localize to the endoplasmic reticulum to initiate apoptosis. J Cell Biol 162: 59-69, 2003.

14. Rao R, Hermel E, Castro-Obregon S, et al: Coupling endoplasmic reticulum stress to the cell death program. Mechanism of caspase activation. J Biol Chem 276: 33869-33874, 2001.

15. Yu S, Andrabi S, Wang $\mathrm{H}$, et al: Apoptosis-inducing factor mediates poly (ADP-ribose)(PAR) polymer-induced cell death Proc Natl Acad Sci USA 103: 18314-18319, 2006.

16. Rao R, Peel A, Logvinova A, et al: Coupling endoplasmic reticulum stress to the cell death program: role of the ER chaperone GRP78. FEBS Lett 514: 122-128, 2002.

17. Lee A: GRP78 induction in cancer: therapeutic and prognostic implications. Cancer Res 67: 3496-3499, 2007.

18. Ogata M, Hino S, Saito A, et al: Autophagy is activated for cell survival after endoplasmic reticulum stress. Mol Cell Biol 26: 9220-9231, 2006.
19. Nakagawa T and Yuan J: Cross-talk between two cysteine protease families: activation of caspase-12 by calpain in apoptosis. J Cell Biol 150: 887-894, 2000.

20. Ron D and Habener J: CHOP, a novel developmentally regulated nuclear protein that dimerizes with transcription factors C/EBP and LAP and functions as a dominant-negative inhibitor of gene transcription. Genes Dev 6: 439-453, 1992.

21. McCullough K, Martindale J, Klotz L, Aw T and Holbrook N: Gadd153 sensitizes cells to endoplasmic reticulum stress by down-regulating $\mathrm{Bcl} 2$ and perturbing the cellular redox state. Mol Cell Biol 21: 1249-1259, 2001.

22. Ueda H, Ullrich SJ, Gangemi JD, et al: Functional inactivation but not structural mutation of p53 causes liver cancer. Nat Genet 9: 41-47, 1995.

23. Susin S, Lorenzo H, Zamzami N, et al: Molecular characterization of mitochondrial apoptosis-inducing factor. Nature 397: 441-446, 1999.

24. Zhang X, Chen J, Graham S, et al: Intranuclear localization of apoptosis inducing factor (AIF) and large scale dna fragmentation after traumatic brain injury in rats and in neuronal cultures exposed to peroxynitrite. J Neurochem 82: 181-191, 2002.

25. Andrabi S, Kim N, Yu S, et al: Poly (ADP-ribose)(PAR) polymer is a death signal. Proc Natl Acad Sci USA 103: 18308-18313, 2006.

26. Reddy R, Mao C, Baumeister P, Austin R, Kaufman R and Lee A: Endoplasmic reticulum chaperone protein GRP78 protects cells from apoptosis induced by topoisomerase inhibitors. J Biol Chem 278: 20915-20924, 2003.

27. Healy SJM, Gorman AM, Mousavi-Shafaei P, Gupta S and Samali A: Targeting the endoplasmic reticulum-stress response as an anticancer strategy. Eur J Pharmacol 625: 234-246, 2009.

28. Pyrko P, Schönthal A, Hofman F, Chen T and Lee A: The unfolded protein response regulator GRP78/BiP as a novel target for increasing chemosensitivity in malignant gliomas. Cancer Res 67: 9809-9816, 2007.

29. Suzuki T, Lu J, Zahed M, Kita K and Suzuki N: Reduction of GRP78 expression with siRNA activates unfolded protein response leading to apoptosis in HeLa cells. Arch Biochem Biophys 468: 1-14, 2007.

30. Dong D, Ni M, Li J, et al: Critical role of the stress chaperone GRP78/BiP in tumor proliferation, survival, and tumor angiogenesis in transgene-induced mammary tumor development. Cancer Res 68: 498-505, 2008.

31. Kouroku Y,Fujita E, Tanida I, et al: ER stress (PERK//eIF2[alpha] phosphorylation) mediates the polyglutamine-induced LC3 conversion, an essential step for autophagy formation. Cell Death Differ 14: 230-239, 2007.

32. Ding W, Ni H, Gao W, et al: Differential effects of endoplasmic reticulum stress-induced autophagy on cell survival. J Biol Chem 282: 4702-4710, 2007.

33. Li J, Hou N, Faried A, Tsutsumi S, Takeuchi T and Kuwano H: Inhibition of autophagy by 3-MA enhances the effect of 5-FU-induced apoptosis in colon cancer cells. Ann Surg Oncol 16: 761-771, 2009.

34. Li J, Hou N, Faried A, Tsutsumi S and Kuwano H: Inhibition of autophagy augments 5-fluorouracil chemotherapy in human colon cancer in vitro and in vivo model. Eur J Cancer 46: 1900-1909, 2010. 